Cross-sectional study

\section{Greater nurse autonomy associated with lower mortality and failure to rescue rates}

10.1136/eb-2016-102591

Catharina van Oostveen, ${ }^{1}$ Hester Vermeulen ${ }^{2}$

${ }^{1}$ Spaarne Gasthuis Academy, Spaarne Gasthuis Hospital, Hoofddorp, The Netherlands; ${ }^{2}$ IQ Healthcare Radboud Institute of Health Sciences, Scientific Center for Quality of Healthcare, Nijmegen, The Netherlands

Correspondence to: Catharina van Oostveen, Spaarne Gasthuis Academy, Spaarne Gasthuis Hospital, PO Box 770, Hoofddorp 2130AT, The Netherlands; cvanoostveen@spaarnegasthuis.nl

Commentary on: Rao AD, Kumar A, McHugh M. Better nurse autonomy decreases the odds of 30-day mortality and failure to rescue. J Nurs Scholarsh 2016;00:1-7.

\section{Implications for practice and research}

- Hospitals are responsible for providing the means necessary for nurses to act autonomously and positively influence patient outcomes.

- To prove causality, the link between nurse autonomy and patient outcomes should be endorsed by using robust research designs, examining results over time to assess differences in autonomy levels.

\section{Context}

There is a growing body of evidence linking professional work environments to improved patient, personnel and organisational outcomes. Since autonomy has been identified as an important attribute of a professional work environment for enhancing patient safety, ${ }^{1}$ a proven association between autonomy and patient-outcomes might be expected but, until now, had not been examined. This study by Rao et al is the first study that links the concept of nurse 'autonomy' in all contexts-clinical autonomy, job autonomy and control over nursing practice-with patient outcomes such as 30-day mortality and failure to rescue (FTR).

\section{Methods}

This study used cross-sectional data from the earlier Multistate Nursing Care and Patient safety Study $(2006-2007)^{2}$ to examine if patient outcomes such as 30-mortality and FTR are better in hospitals where nurses report greater levels of autonomy. The sample consisted of 100000 randomly sampled registered nurses from four states in the USA (California, Florida, Pennsylvania and New Jersey). They were surveyed for a range of measures such as the Nursing Workload Index-Revised (NWI-R), from which an autonomy subscale was created. Additionally, data were collected to characterise the hospitals in which these nurses worked, and patient characteristics and outcomes of patients who received care in these hospitals. Logistic regression models, including a set of covariates to account for nursing (staffing, education), hospital (size, teaching status) and patient characteristics (diagnosis, comorbidity), were used to examine the relationship between autonomy and outcomes.

\section{Findings}

Reported autonomy levels varied across hospitals. For example, nurses employed in small $(<100$ beds) and non-teaching hospitals reported the highest levels of autonomy scores. More importantly, each additional point on nurse autonomy was associated with 19\% lower odds of 30-day mortality and 17\% lower odds of FTR. These findings suggest that patients receiving care within hospitals that promote nurse autonomy have lower risk for death within 30 days and complications leading to death within 30 days.

\section{Commentary}

Despite the observational nature and use of secondary analysis, the study is meticulously designed and the results are in line with earlier research linking positive work environments with higher levels of autonomy to improved patient outcomes. ${ }^{3}$ The authors address some limitations of the design. We would add that general attitudes towards work environment may influence individual item responses. Furthermore, relying on surveyed nurses to establish an overall census of bachelor nurses and nurse staffing may be imprecise. ${ }^{4}$ To draw more causal conclusions, the link between nurse autonomy and patient outcomes should be endorsed by using robust research designs-that is, time series designs-examining results over time to assess differences within hospitals based on variations in autonomy levels.

The fact that Rao et al operationalised 'autonomy' for the full breadth of the concept means that their results express the value of nurses' clinical knowledge in decision-making for improving patient outcomes. Furthermore, it highlights the importance of empowerment-in terms of enhancing nurse autonomy-of the largest workforce within hospitals.

This study provides evidence that when nurses do not have the ability to exercise their clinical and organisational knowledge, patient safety is put at risk. Therefore, healthcare organisations are responsible for providing necessary means for nurses to act autonomously, that is, by formulating clear roles, responsibilities and behaviours; enhancing competence in practice and decision-making; using shared governance organisational structures, and by creating strong visible leadership. However, nurses should also take responsibility for their own behaviours and actions. 'To optimise its value, autonomy must be accompanied by proactive behaviour (ie, self-starting and forward thinking to prevent, rather than react to, workplace problems) on the part of nurses,. ${ }^{5}$

Twitter Follow Catharina van Oostveen@CathvanOostveen

Competing interests None declared.

Provenance and peer review Commissioned; internally peer reviewed.

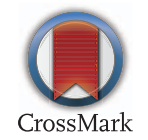

\section{References}

1. Institute of Medicine. Keeping patients safe: transforming the work environment of nurses. National Academy Press, 2004.

2. Aiken LH, Cimiotti JP, Sloane DM, et al. Effects of nurse staffing and nurse education on patient deaths in hospitals with different work environments. Med Care 2011;49:1047-53.

3. Aiken LH, Clark SP, Sloane DM, et al. Effects of hospital care environment on patient mortality and nurse outcomes. J Nurs Adm 2008;38:223-9.

4. Needleman J. Nursing skill mix and patient outcomes. BMJ Qual Saf 2016. doi:10.1136/bmjqs-2016-006197 [Epub ahead of print 30 Dec 2016].

5. Stalpers D. Effective excellence in nursing; bridging the gap between measurement of quality of nursing care and clinical reality. University of Utrecht, 2016. 